\title{
Batch Training for Neuromorphic Systems with Device Non-idealities
}

\author{
Yutong Gao \\ George Washington University, \\ Computer Science Department \\ Washington, D.C. \\ gao418@gwu.edu
}

\author{
Shang $\mathrm{Wu}$ \\ George Washington University, \\ Computer Science Department \\ Washington, D.C. \\ swu23@gwu.edu
}

\author{
Gina C. Adam \\ George Washington University, \\ ECE Department \\ Washington, D.C. \\ ginaadam@email.gwu.edu
}

\begin{abstract}
Neuromorphic hardware based on emerging analog memory technologies, like resistive switching, show promise for dense energy efficient systems given their ultra-scalable footprint and better energy / bit consumption. Training of neuromorphic systems large enough for useful real-life applications is theoretically highly efficient, however it poses significant practical challenges due to device non-idealities, such as stochasticity, asymmetry, and nonlinearity.

We investigate in detail mini-batch gradient descent in the context of device non-idealities using a 3-layer MLP on MNIST. Convergence curves for different batch sizes show a consistent increase in performance with the increased batch size, but is highly sensitive to the learning rate. Statistical analysis of the weight trajectories and histograms show different behavior between the software and the non-ideal hardware case, but further investigations are needed to understand the trends in the weight populations across the training for different batch sizes.
\end{abstract}

\section{CCS CONCEPTS}

- Hardware; • Computer systems organization $\rightarrow$ Neural networks;

\section{KEYWORDS}

Resistive Switching, RRAM, Memristor, Non-ideal, Neural Network, Batch Training, Mini-Batch Gradient Descent

\section{ACM Reference Format:}

Yutong Gao, Shang Wu, and Gina C. Adam. 2020. Batch Training for Neuromorphic Systems with Device Non-idealities. In International Conference on Neuromorphic Systems 2020 (ICONS 2020), July 28-30, 2020, Oak Ridge, TN, USA. ACM, New York, NY, USA, 4 pages. https://doi.org/10.1145/3407197. 3407208

\section{INTRODUCTION}

As high performance computing hardware like GPUs and TPUs have increased exponentially in recent years, deep artificial neural network structures regarded as unachievable a few years ago, are

Permission to make digital or hard copies of all or part of this work for personal or classroom use is granted without fee provided that copies are not made or distributed for profit or commercial advantage and that copies bear this notice and the full citation on the first page. Copyrights for components of this work owned by others than the author(s) must be honored. Abstracting with credit is permitted. To copy otherwise, or republish, to post on servers or to redistribute to lists, requires prior specific permission and/or a fee. Request permissions from permissions@acm.org.

ICONS 2020, July 28-30, 2020, Oak Ridge, TN, USA

(C) 2020 Copyright held by the owner/author(s). Publication rights licensed to ACM. ACM ISBN 978-1-4503-8851-1/20/07...\$15.00

https://doi.org/10.1145/3407197.3407208 now routinely investigated. However, training of such neural networks is expected to consume significant computing resources in the coming decades at large environmental and financial costs [12].

Exploiting new analog memory technologies, such as resistive switching devices (ReRAM) and phase change memory (PCM), promises to accelerate DNN training by eliminating this computingmemory bottleneck. For inference operations, traditional computer models based on stochastic gradient descent (SGD) can be used to determine the weight values of the model offline, which can then be programmed into the hardware analog arrays. Matrix multiplication operation maps physically to Kirchoff's law allowing for a single read operation with great efficiency. [4].

On the other hand, online training of such hardware networks using SGD poses significant practical challenges due to device nonidealities, including device variability and nonlinearity in the weight update [1]. Such device non-idealities, particularly in larger scale networks, can lead to poor performance or even non-convergence $[2,3]$. Research has suggested that the requirements on device performance are extremely tight, but are also highly dependent on the algorithm. For example, Kataeva et al. [7] show that online stochastic gradient descent training modeled on a system with $2 \%$ device nonlinearity and asymmetry used for MNIST training leads to almost $20 \%$ miss-classification. By comparison, batch training implemented using the Manhattan update rule, shows much better performance of $1.5 \%$ misclassification. While devices of superior performance and uniformity are being investigated, alternative training algorithms resilient to device non-idealities are an active area of research. Our contributions are:

- We have investigated batch training in the context of neural network training SGD and mini-batch gradient descent (MBGD) with modeled device non-idealities. The non-idealities include a finite resistance range, weight update asymmetry, weight update nonlinearity, and stochasticity.

- Our investigation has shown that increasing the batch size can lead to an absolute $35-40 \%$ increase in accuracy on a one hidden layer network for MNIST dataset, but is highly sensitive to the learning rate.

- Preliminary results of the weight statistical properties show that the weight trajectories and distributions are different in the hardware model than in the software case, despite a seemingly similar accuracy convergence pattern.

The remainder of the paper is organized as follows. Section II describes the device models and our detailed algorithmic implementation. Section IV presents the evaluation of the batch training on MNIST dataset using devices with non-idealities vs. ideal device models. Section V concludes with a final discussion. 


\section{METHODS}

\subsection{Device models}

A resistive switch (or memristor or ReRAM) is a passive electronic device based on a metal-insulator-metal stack, typically in a twoterminal crosspoint structure. After an initial "forming" step to create a conductive filament, the device can be programmed repeatedly to a desired state in the OFF (low) - ON (high) conductance range using low amplitude voltage pulses to modulate the shape of the filament.[5, 13]. However due to their physics of switching in amorphous materials and manufacturing challenges, this technology suffers from significant practical challenges due to device non-idealities, such as stochasticity, asymmetry, and nonlinearity

To model these non-idealities for batch training, we started with the useful open access NeuroSim plaftorm developed by S. Yu [10] This paper uses an analog eNVM (the non-ideal device) model and an ideal device model. The two device models used are is shown in Fig. 1. The ideal device model assumes reproducible behavior with no stochasticity and a linear relationship between the applied number of pulses and the obtained conductance state as seen in Fig. 1a. In the non-ideal device model, there is 1) non-linearity between the applied pulses and the conductance which leads to imperfect weight programming 2) asymmetry between the set (potentiation) and reset (depression) and 3) variability in the operation This stochasticity is sufficiently large that sending an "increase weight" pulse can even randomly lead to a "decreased weight" and vice versa (Fig. 1b).
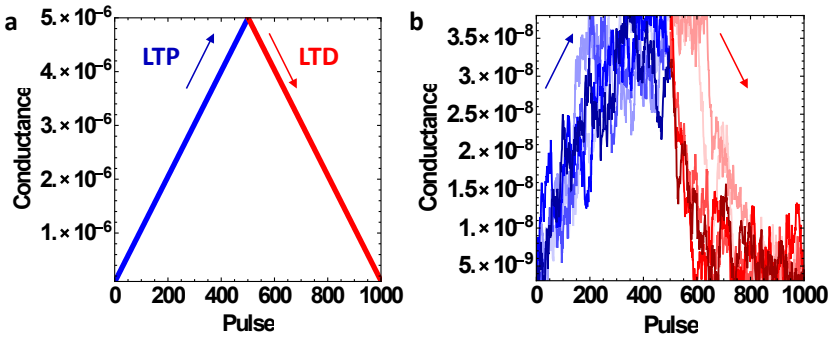

Figure 1: Device models. a) Ideal device with linear weight update, large ON / OFF ratio, no asymmetry and no device variability; b) Non-ideal device model with smaller ON / OFF ratio, weight update asymmetry, weight update nonlinearity, and cycle-to-cycle variability ( 5 cycles shown).

\subsection{Multilayer Perceptron}

To investigate the impact of batch training on networks with nonideal weights, a 3-layer multi-layer perceptron (MLP) has been used (Fig. 2). The vector $X$ is the data input applied to the neurons in the input layer, which are fully connected to the neurons in the hidden layer via a synaptic connection weight matrix $W$. By multiplying the input vector $X$ with the weight matrix $W$, the output vector $H$ of the hidden layer is obtained. Likewise, the output vector of the hidden layer can be propagated forward through the weight matrix between hidden layer and output layer to obtain the output result $Y$. To train the network, the error is generated from the ideal output result $Z$ and the actual output result $Y$. The calculated error

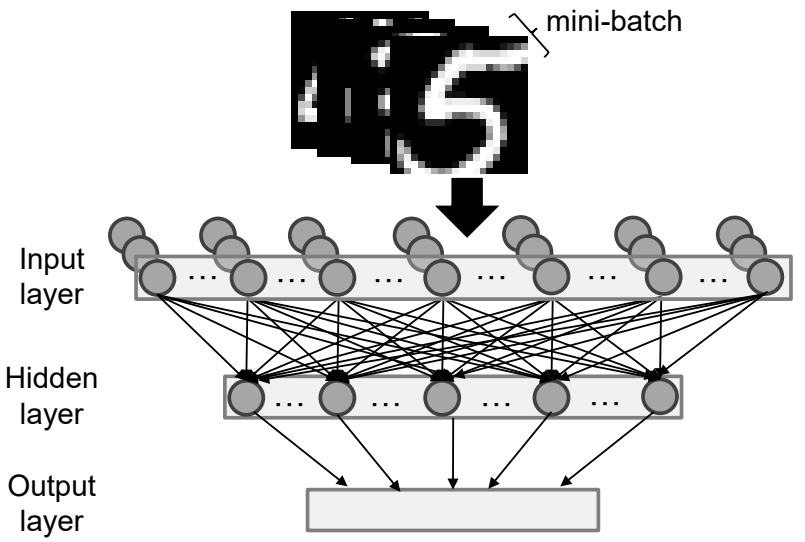

Figure 2: Network model showing a three layer perceptron of size $400 \times 100 \times 10$ for MNIST batch training.

is backpropagated through the network and the weight matrix values between each layer are updated by using gradient descent to minimize the error of the final output. In practice, the $\Delta W$ are calculated using a stochastic approximation of the gradient, over a sample inputs of batch size $B$. In MLP models based on memristor synapses, the values of the weight matrices can be stored in memristor crossbars witch each conductance of the resistive switch used to represent the weight of a synaptic connection $[8,11]$.

\subsection{Mini-batch Gradient Descent}

Traditional stochastic gradient descent (SGD) randomly selects one image in the dataset to calculate the gradient and update the weights, then repeat it for all the examples in the training dataset. Since only one input point is used per iteration, the weights have to be updated often which makes the system susceptible to nonidealities. By comparison, mini-batch gradient descent (MBGD) uses a randomly selected batch of samples of size $B$ to calculate a mean gradient and update the weights, then repeat for all the mini-batches in the dataset.

For this work, we have updated the NeuroSim v3.0 to include the traditional MBGD algorithm in addition to its other algorithmic implementations [10]. NeuroSim 3.0 has its own unique versions of momentum, Adagrad, RMSprop, and Adam, but not MBGD.

It's important to note, however, since real devices cannot linearly integrate gradients, different possible implementations of MBGD exist. After generating a batch of samples, one could sequentially update the array with each batch member's input activations and errors without first summing up the gradients. This eliminates the need for an external memory array to store the fully recomposed gradient. Hoskins et al. [6] called this MBGD1. In contrast, we implemented a different version, MBGD2, where the gradient is first summed prior to updating the matrix. This reduces the number of device updates on the array by a factor of $B$ (the batch size) per cycle and reduces the uncertainty in the gradient. As mentioned in [6], while the two methods are equivalent in software, gradient calculation in the context of training on non-ideal platforms can be very sensitive to the order of operations. Our preliminary investigations on MBGD1 had poor training performance. For the 
remaining of the paper, we focus on the MBGD2 implementation. In addition, to speed up the training, our implementation of MBGD was parallelized with each mini-batch divided into $k$ partitions to be able to use $k$ machines (or threads) running in parallel. For each element in the partition, the gradient is calculated on its respective machine (or thread), then their results are averaged to get the mean gradient [9]. This acceleration was necessary in order to efficiently implement the learning rate optimizations needed for the results.

\section{RESULTS}

Our investigation has shown that increasing the batch size can lead to an absolute $35-40 \%$ increase in accuracy for the 3-layer network with non-ideal device model for the MNIST dataset by comparison with the standard SGD, as shown in Fig. 3. As expected, the ideal device model performs very close to the software counterpart for the SGD, while the non-ideal device model has a poor performance with an accuracy $51 \%$ and a very noisy convergence pattern (Fig. 3a). By adopting the method of mini-batch training, the errors caused by the read and write operations in the device during the weight update can be averaged out. Compared with SGD, this method significantly increases the test accuracy to $86 \%$ for batch size $=128$. Moreover the converged curve is much smoother. This is expected since the gradient of each back-propagation update is the result of averaging the gradient of each instance in the mini-batch. The accuracy for the ideal device model slightly decreases from its SGD counterpart, probably due to the fact that the mini-batch algorithm reduces the randomness in the training (Fig. 3b).

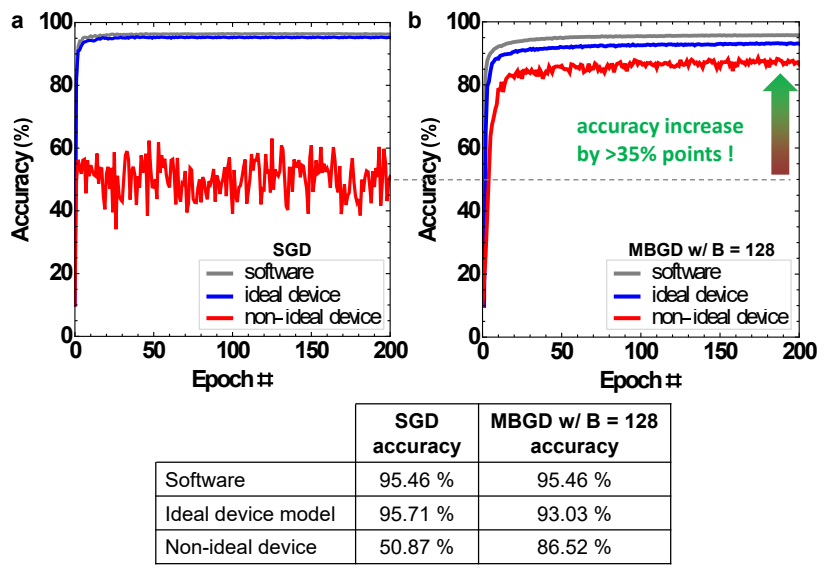

Figure 3: Test accuracy results for the software vs. ideal device model vs. non-ideal device model. a) for SGD; b) for MBGD with batch size $=128$. A large improvement in accuracy of $35 \%$ points and a smoother convergence curve is observed in comparison with the SGD case.

The results in Fig.3 have been obtained with optimized learning rate. Hyperparameter optimization seems to be particularly important for batch training with non-ideal devices. For example, Fig. 4 shows the learning rate (LR) optimization, with the LR window of high accuracy shrinking at higher batch sizes. The optimum LR seems to have a somewhat linear increase with the batch size. The LR and other hyperparameter optimization are critical for understanding how algorithms impact with non-ideal devices. The
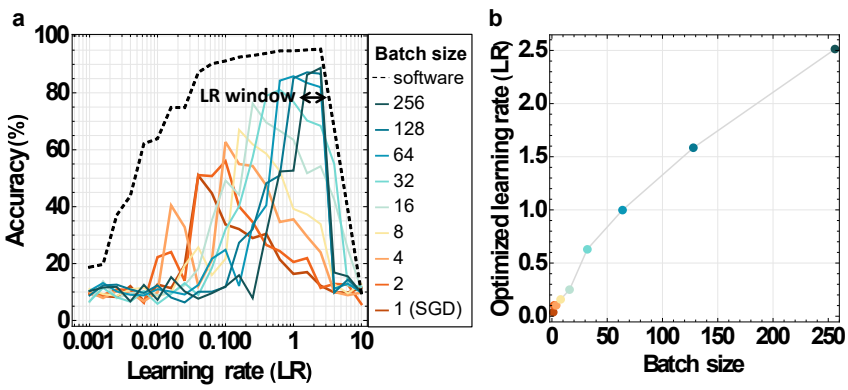

Figure 4: Learning rate (LR) optimization. (a) Accuracy vs. learning rate. Optimized LR plays a critical role in achieving high accuracy, particularly at larger batch sizes when the LR window shrinks. b) LR vs. batch size showing that the LR seems to have a somewhat linear increase with the batch size. All results are shown for the non-ideal device model.

software model converges over a wide range of learning rates. In contrast, naively changing the batch size without changing the LR in the non-ideal device case may lead one to believe that increasing the batch size decreases performance rather than increases it. For example, increasing the batch size from 1 (SGD) to 128 with a learning rate of 0.1 causes the accuracy to fall from $50 \%$ to $10 \%$.

Fig. 5 summarizes the investigation of convergence vs. accuracy vs. batch size for the non-ideal device model. The accuracy consistently increases with the batch size and the convergence curve becomes smoother (Fig. 5a). This is expected as the size of $B$ determines how smooth the calculated gradient is between adjacent iterations. Significant improvement in accuracy is obtained even at lower batch sizes, such as $\mathrm{B}=32$ (accuracy $=81 \%$ ), as shown in Fig. 5b. However, accuracy trends shown in Fig. 5c predict that softwarelevel accuracy $95 \%$ can be obtained at B $=4096$. However, more investigation is needed since update quantization makes handling small gradients difficult, and an alternative approach to handling small gradients may improve the large batch size performance.

In [10], a $40 \%$ to $50 \%$ improvement in accuracy was achieved using the Adam training algorithm which integrates the gradient over prior history and uses an adaptive learning rate unique to each variable. While that approach is not especially scalable, our results show that gradient integration and proper choice of the learning rate could be the only prerequisites for improving the accuracy.

An investigation of the weight trajectories and a histogram comparison between software and non-ideal hardware case highlights that the mechanisms behind convergence in the two cases could be significantly different, although their convergence curve looks similar. For the software case, the trajectories of 10 randomly picked weights are smooth and uniformly converging, while the weight distributions are bimodal for both SGD and batch $=128$ case as shown in Fig. 6a. By comparison, the non-ideal device model shows a seemingly random pattern in the weight trajectories for both SGD and batch $=128$, although the batch training case reaches $86 \%$ accuracy after 200 epochs of training. The weight distribution is skewed towards negative values and wider for the batch training case. $\mathrm{Fu}-$ ture statistical investigations into the correlation matrices of these weights might help elucidate the mechanisms behind the improved batch training performance in the context of device non-idealities. 

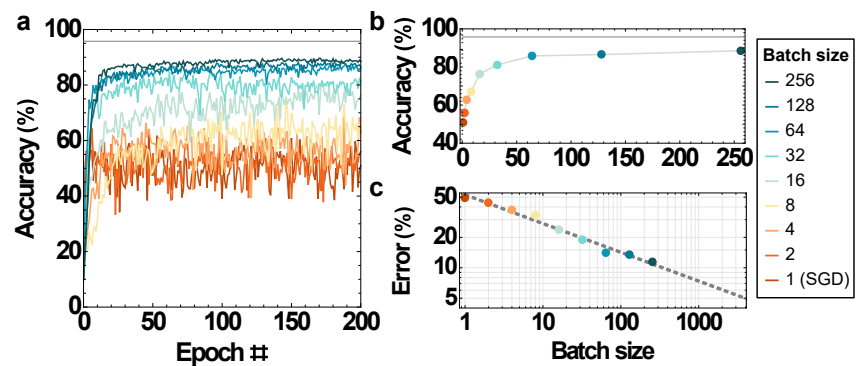

Figure 5: Batch training results for the non-ideal device model. (a) Convergence curves for different batch sizes. The convergence is smoother and less noisy with increased batch size. (b) Accuracy vs. batch size, showing consistent increase in performance with increased batch size from $50 \%$ accuracy for the SGD case to $89 \%$ at batch size $=256$. The optimized LR from Fig. 4 was used for each batch size result. (c) Accuracy trends for the non-ideal device model training at higher batch sizes. At batch size $=4096$, it is predicted to reach software-level accuracy of $95 \%$.

\section{CONCLUSION}

In this paper, we have investigated in a detailed fashion the circumstantial evidence from the literature that mini-batch training can overcome the negative impact of device non-idealities. Our investigation has shown that increasing the batch size can lead to an absolute $40 \%$ increase in accuracy on a one-hidden layer network based on non-ideal devices trained for batch size $=256$ on MNIST dataset. Exploring different device models with different non-idealities profiles and applying this methodology to more complex networks would lead to additional insight into mini-batch gradient descent as a useful training methodology for emerging non-volatile neuromorphic hardware.

\section{ACKNOWLEDGMENTS}

The authors would like to thank Xibing Chen, Siyuan Huang and the NIST Alternative Computing Group for useful scientific discussions. This work has been supported by the DARPA / ONR grant No. N00014-20-1-2031 and GWU University Facilitating Fund.

\section{REFERENCES}

[1] Gina C. Adam, Ali Khiat, and Themis Prodromakis. 2018. Challenges hindering memristive neuromorphic hardware from going mainstream. Nature Communications 9,1 (2018)

[2] Stefano Ambrogio, Pritish Narayanan, Hsinyu Tsai, Robert M. Shelby, Irem Boybat, Carmelo Di Nolfo, Severin Sidler, Massimo Giordano, Martina Bodini, Nathan C. P. Farinha, and et al. 2018. Equivalent-accuracy accelerated neural-network training using analogue memory. Nature 558, 7708 (2018), 60-67.

[3] Farnood Merrikh Bayat, Mirko Prezioso, Bhaswar Chakrabarti, Irina Kataeva, and Dmitri Strukov. 2017. Memristor-based perceptron classifier: Increasing complexity and coping with imperfect hardware. 2017 IEEE/ACM International Conference on Computer-Aided Design (ICCAD) (2017), 549-554.

[4] L. Ceze, J. Hasler, K. K. Likharev, J.-S. Seo, T. Sherwood, D. Strukov, Y. Xie, and S. Yu. 2016. Nanoelectronic neurocomputing: Status and prospects. 2016 74th Annual Device Research Conference (DRC) (2016)

[5] M. Di Ventra, Y.V. Pershin, and Chua Leon. 2009. Circuit elements with memory: memristors, memcapacitors, and meminductors. Proceedings of IEEE 97(10), 1717-1724 (2009).

[6] Brian D. Hoskins, Matthew W. Daniels, Siyuan Huang, Advait Madhavan, Gina C. Adam, Nikolai Zhitenev, Jabez J. Mcclelland, and Mark D. Stiles. 2019. Streaming
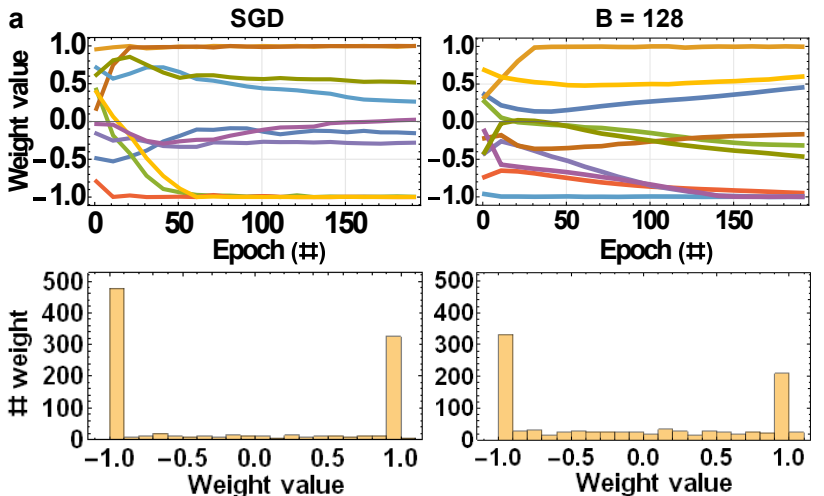

b
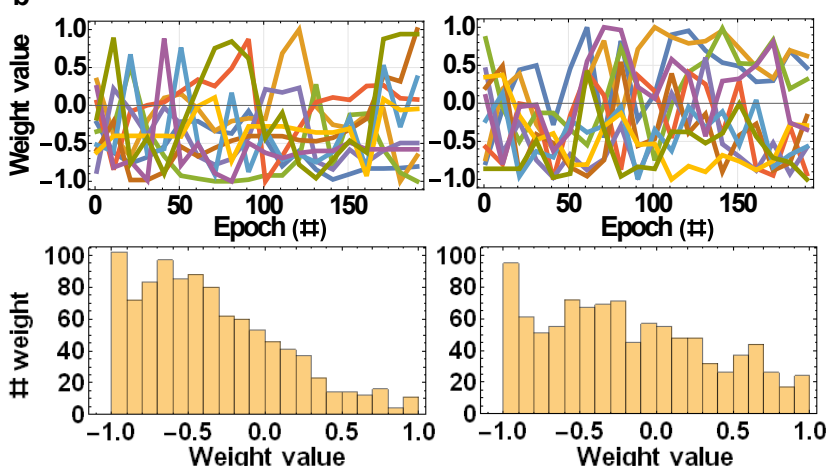

Figure 6: Weight trajectories and histograms. (a) for software model with SGD and batch $=128$. Trajectories for 10 random weights show smooth convergence. The weight histograms have a bimodal distribution with weight values aggregated around -1 and 1 values due to an imposed truncation in the $[-1,1]$ interval. (b) for non-ideal device model with SGD and batch $=128$. The weight trajectories show random behavior. The histograms show a distribution skewed towards negative values, wider for the MBGD case. Results shown for the hidden-to-output layer, similar results for input-to-hidden.

Batch Eigenupdates for Hardware Neural Networks. Front. Neurosci. 13 (793) (2019).

[7] Irina Kataeva, Farnood Merrikh-Bayat, Elham Zamanidoost, and Dmitri Strukov. 2015. Efficient training algorithms for neural networks based on memristive crossbar circuits. 2015 International foint Conference on Neural Networks (IFCNN) (2015).

[8] Can Li, Daniel Belkin, Yunning Li, Peng Yan, Miao Hu, Ning Ge, Hao Jiang, Eric Montgomery, Peng Lin, Zhongrui Wang, and et al. 2018. Efficient and self-adaptive in-situ learning in multilayer memristor neural networks. Nature Communications 9, 1 (2018)

[9] Mu Li, Tong Zhang, Yuqiang Chen, and Alexander J. Smola. 2014. Efficient minibatch training for stochastic optimization. Proceedings of the 20th ACM SIGKDD international conference on Knowledge discovery and data mining - KDD 14 (2014).

[10] Yandong Luo, Xiaochen Peng, and Shimeng Yu. 2019. MLP+ NeuroSimV3. 0: Improving On-chip Learning Performance with Device to Algorithm Optimizations. Proceedings of the International Conference on Neuromorphic Systems (2019).

[11] M. Prezioso, F. Merrikh-Bayat, B. D. Hoskins, G. C. Adam, K. K. Likharev, and D. B. Strukov. 2015. Training and operation of an integrated neuromorphic network based on metal-oxide memristors. Nature 521, 7550 (2015), 61-64.

[12] Emma Strubell, Ananya Ganesh, and Andrew Mccallum. 2019. Energy and Policy Considerations for Deep Learning in NLP. Proceedings of the 57th Annual Meeting of the Association for Computational Linguistics (2019).

[13] R. Waser, R. Dittimann, G. Staikov, and K. Szot. 2012. Redox-based resistive switching memories-nanoionic mechanisms, prospects, and challenges. Advanced Materials 21(25-26), 2632-2663 (2012). 